Proc. XIX International School of Semiconducting Compounds, Jaszowiec 1990

\title{
HOT ELECTRON BALLISTIC TRANSPORT IN TWO DIMENSIONAL STRUCTURES
}

\author{
A. Palevski* , U. Sivan, M. Heiblum, C. P. Umbach and H. Strickman \\ IBM Research Division, T. J. Watson Research Center, Yorktown Heights, NY 10598, \\ USA
}

(Received August 8, 1990)

\begin{abstract}
Quasi ballistic transport in a 2 dimension electron gas was explored and tunneling through an electrostatic barrier induced by metallic gates on the top of the heterostructure was demonstrated. For energies above $36 \mathrm{meV}$ we observed the LO phonon emission, leading to a short mean free path. At energies below the phonon energy we find a mean free path of the order of $2 \mu \mathrm{m}$, about an order of magnitude longer than expected theoretically for electron interactions. The angular distribution of hot electrons injected from a point source was shown to be collimated and could be steered by controling the electrostatic profile of the injector. In addition we demonstrated operation of electrostatic lens which opens a new branch in semiconductor physics, namely, electron- optics in solids.
\end{abstract}

PACS numbers: 73.50.Fq; 73.50.Gr

\section{Introduction}

Quantum mechanical principles are playing increasingly important role in today's design of solid state devices, such as resonant tunneling diodes, hot electron ballistic transistors, etc. [1]. The small dimensions needed for realization of quantum transport in mentioned structures until recently were achieved in vertical direction only (the direction perpendicular to the plane of epitaxial layers).

The ability to fabricate structures with lateral dimensions comparable to electron elastic and/or inelastic mean free path ( $\mathrm{mfp}$ ) allows to observe spectacular effects such as Aharonov-Bohm oscillations of conductance, universal conductance fluctuation, magnetic focusing of electron, quantized ballistic resistance, lateral hot electron ballistic transport, electrostatic collimation and focusing and many other

*Present address: AT\&T Bell Laboratories, Murray Hill, New Jersey 07974, USA. 
effects exhibiting the quantum nature of electronic transport in lateral domain [2]. Here, we present a variety of recent experiments demonstrating realization of lateral hot electron ballistic transport [3] observation of phonon emission [4,5] and electrostatic collimation and focusing [6] of electronic beam in 2D electron gas.

The study of hot electron ballistic transport, [3,4] was done employing a novel three terminal device, whose generic structure is shown in Fig.1a. Two metal-

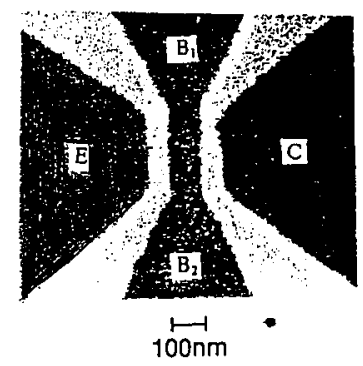

(a)

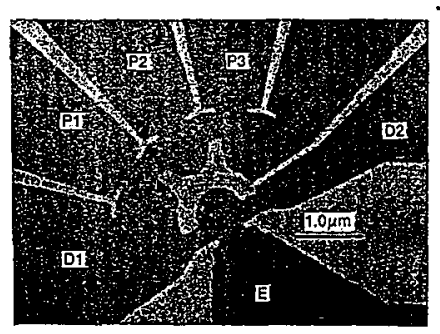

(d)

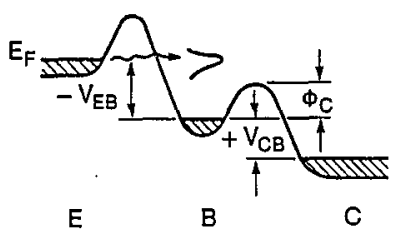

(b)

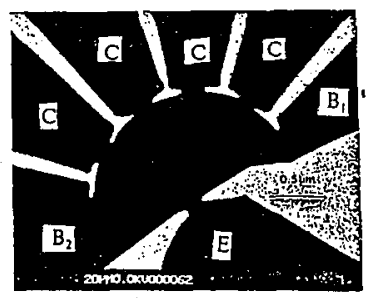

(c)

Fig.1. (a) An SEM micrograph showing the gates configuration of the device used to establish ballistic transport and tunneling through an induced barrier in the 2DEG. (b) A schematic description of the potential distribution for a biased device, $V_{E B}<0$ and $V_{C B}>0$. (c) An SEM micrograph of the device used to investigate the angular distribution of hot electrons injected from a point source. (d) SEM micrograph of electrostatic lens.

lic gates (light areas in Fig.1a) were defined using electron beam lithography on the surface of a $\mathrm{GaAs}-\mathrm{Al}_{0.3} \mathrm{Ga}_{0.7} \mathrm{As}$ heterostructure containing a 2DEG (in the heterojunction between GaAs and AlGaAs). One gate was utilized to produce a barrier for use as a hot electron emitter $(E)$ and the second one was used to produce a spectrometer (or collector) barrier for analyzing the energy distribution of the collection beam (C). The metallic gates were usually $500 \AA$ wide (from left to right), the emitter gate length (top to bottom) varied between $0.25 \mu \mathrm{m}$ and $1.0 \mu \mathrm{m}$, and the collector gate length varied between $0.75 \mu \mathrm{m}$ and $1 \mu \mathrm{m}$. The separation between the emitter and the collector, the base region, ranged in the 
various devices between $50 \mathrm{~nm}$ and $170 \mathrm{~nm}$. The various $2 D E G$ regions, namely $E, B_{1}, B_{2}$, and $C$ were contacted by standard NiGeAu alloyed ohmic contacts. The carrier density and mobility of the different 2DEG used in the various experiments were measured at $4.2 \mathrm{~K}$ using standard van der Pauw procedure and were

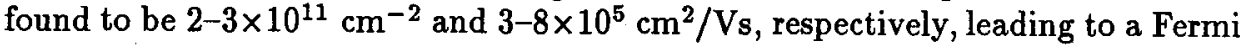
energy of $E_{F} \approx 7-11 \mathrm{meV}$ and a transport mean free path (for cold electrons) of $l \approx 2.5-4.5 \mu \mathrm{m}$.

An application of negative gate voltage with respect to the 2DEG in the base forms a potential barrier under each gate separating thus the emitter and collector regions from the base (Fig.1b). Hot electrons were injected by applying voltage across the emitter barrier $\left(V_{E B}\right.$ in Fig.1b). The collector barrier height was controlled both by changing the collector gate voltage and by changing the collector biasing relative to the base ( $V_{C B}$ in Fig.1b). Monitoring the resistance between two base contacts, $B_{1}$ and $B_{2}$, on both sides of the base we ensured that the base was not depleted of carriers for gate voltages used in the experiment. Energy spectroscopy of injected electrons was performed by measuring the collected current as a function of the collector barrier height. Since tunneling through the collector barrier is negligible, only hot electrons with longitudinal (i.e. perpendicular to the collector) energy larger than the collector barrier height are collected. Electrons which relax to energies below the collector barrier height are collected at the base. This technique constitutes an almost unique way for discriminating non-ballistic transport against a ballistic one. A somewhat more elaborated version of this device [5] (Fig.1c) was utilized to investigate the spatial distribution of the injected electrons and finally Fig.1d shows electrostatic lens used for electron beam focusing.

In the next section we describe a series of experiments establishing directly, via energy spectroscopy of the transmitted electrons, ballistic transport over distances as long as $0.5 \mu \mathrm{m}$. In the section 3 we show that the main scattering mechanism at $4.2 \mathrm{~K}$, for hot electrons with excess energy larger than the longitudinal optical (LO) phonon energy ( $\hbar \omega_{\text {LO }}=36 \mathrm{meV}$ ) is LO phonon emission. For electrons with energy below $36 \mathrm{meV}$ we demonstrate a surprisingly long inelastic $\mathrm{mfp}$ of the order of $2 \mu \mathrm{m}$. Finally, we shall discuss the angular distribution of electrons injected from a point source in 2DEG and demonstrate steering and focusing of hot and cold electrons by controlling the electrostatic profile of the emitter [5] and by putting the electrostatic lens [6] in the transport region between emitter and collector.

\section{Ballistic motion of hot electron}

Typical results for the collector and emitter currents ( $I_{C}$ and $I_{E}$, respectively) vs. the injection voltage $V_{E B}$, for various collector barrier heights, are depicted in Fig.2. At small injection voltages $I_{E}$ is exponential in $V_{E B}$ implying tunneling through the emitter barrier (further support will be supplied later). Although the emitter current is substantial for $\left|V_{E B}\right|>8 \mathrm{mV}$, the collector current is turned on only when $e V_{E B}$ exceeds the collector barrier height $\Phi_{C}$, indicating ballistic 


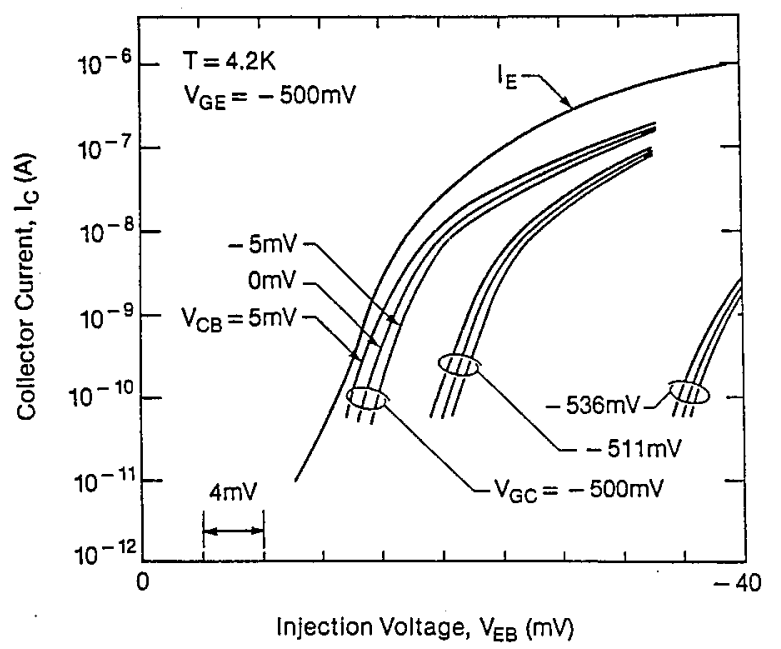

Fig.2. The collector current, $I_{C}$, as a function of the injection voltage, $V_{E B}$, when the collector-gate voltage, $V_{G C}$, and the collector-base voltage, $V_{C B}$, serve as parameters. The current onset, indicative of the collector barrier height, is strongly influenced by both parameters. The injected current, $I_{E}$, is independent of these parameters

transport (the collector barrier height is tuned here by varying both the collector gate voltage and $V_{C B}$ ). Figure 3 a (solid lines) shows a typical family of $I_{C}$ vs. $V_{C B}$ characteristics for gates voltages $V_{G E}=V_{G C}=-0.5 \mathrm{~V}$ and different injection energies, all chosen to be higher than the unbiased spectrometer barrier height (for $V_{E B}=0 \mathrm{~V}, I_{C}=0$ in the range of interest). For $V_{C B}>0$, the collector current increases only slowly as $V_{C B}$ is increased, suggesting that most of the hot electrons have energies higher then the unbiased potential barrier height. When the polarity of $V_{C B}$ is reversed, leading to an increase in $\Phi_{C}$, the collector current decreases slowly initially, followed subsequently by a sharp drop to zero over a range of a few $\mathrm{mV}$. This behavior indicates that electrons with a narrow energy distribution are cut-off by the spectrometer barrier. Note the absence of a 'knee' observed for the lower injector barriers heights indicating that the injected distributions are broad, extending from the injection energy $e V_{E B}$ and below, and do not result from tunneling. For a low injector barrier, the application of an injection voltage lowers the barrier below the Fermi energy in the emitter.

The number of electrons arriving at the collector at a given energy can be shown to be proportional to $\mathrm{d} I_{C} / \mathrm{d} \Phi_{C}$ at that energy. Given the correspondence between $V_{C B}$ and $\Phi_{C}$ (which can be deduced from the onset of $I_{C}$ in Fig.2) one can derive the energy profile of the collected beam from the data presented in Fig.3a. The results of this analysis are presented in Fig.3b. The energy distributions have a full width at half maximum of about $5 \mathrm{meV}$, which tends to increase as the injection energy is increased (up to $10 \mathrm{meV}$ for $e V_{B E}=30 \mathrm{mV}$ (not shown)). The peaks of the distributions shift with the injection energy (except the lowest one 

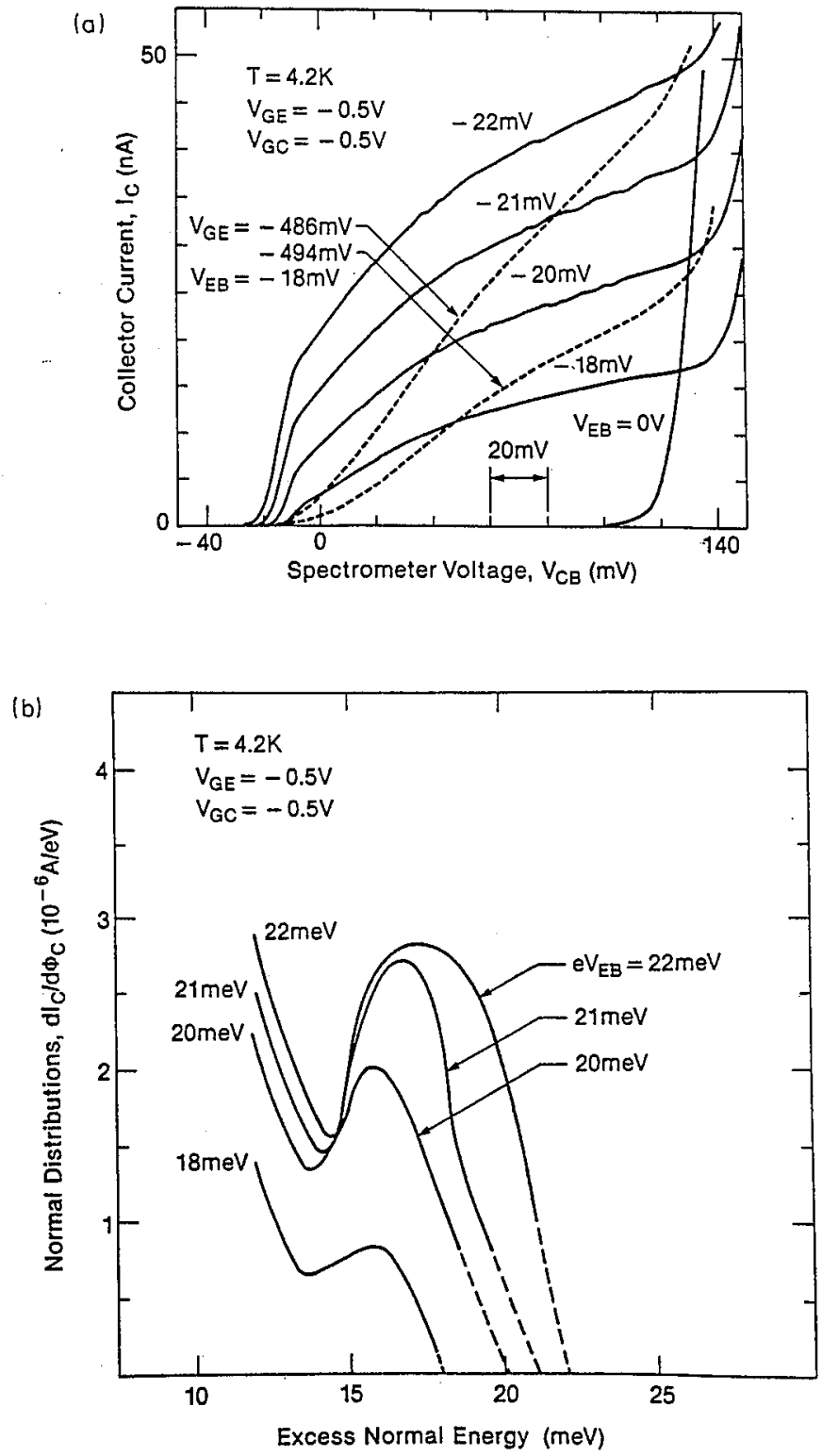

Fig.3. (a) The family of the collector current characteristics, where $V_{E B}=0,-18$, $-20,-21,-22 \mathrm{mV}$. Note the sharp rise in the current (for $V_{C B}=10 \mathrm{mV}$ ) followed by a moderate rise indicative of a narrow ballistic distribution. The doted lines are for lower injector barrier heights and for $e V_{E B}=-18 \mathrm{mV}$, where the injected distributions are very broad. (b) The ballistic distributions for different injection energies as a function of excess normal energy above the Fermi level in the base. The peaks of the distributions follow rigidly the injection energy. At higher $\left|V_{C B}\right|$ leakage currents dominated. 
which is obscured by the lower energy tail) as expected in ballistic transport. The peak energies which are lower by about 3-4 meV than the corresponding Fermi level in the emitter (noted by the crossing of the dotted lines with the energy scale), and the narrow distribution width $(\sim 5 \mathrm{meV})$, are both expected for normal energy distributions injected by $50 \mathrm{~nm}$ wide $20 \mathrm{meV}$ high tunnel barriers. These results prove that tunneling is the injection mechanism in our induced barriers. Even though small angle elastic scattering events can not be excluded, the narrow width of the electron distributions strongly suggest that elastic scattering in our lateral structures is minimal.

The ballistic fraction, $\alpha$, is defined as the ratio between the number of collected ballistic electrons and the total number of injected electrons (the injected current $I_{E}$ ). From Fig.3a we find $I_{C} / I_{E} \approx 0.25$ at $V_{C B}=0$, which is approximately the ballistic fraction collected. Since some of the injected current emerged from the periphery of the injector barrier and never surmounted the spectrometer barrier, the value 0.25 does not reflect the true ballistic mean free path $(\lambda)$. To minimize the number of these 'stray' electrons, different structures where the collector gate was three times longer $(0.75 \mu \mathrm{m})$ than the emitter gate $(0.25 \mu \mathrm{m})$ were made (base width $170 \mathrm{~nm}$ ), and ballistic fractions higher than 0.7 were measured. Using $\alpha=\exp \left(-d_{B} / \lambda\right)=0.7$, where $d_{B}$ is the effective base width for hot electrons, we find $\lambda=480 \mathrm{~nm}$. This lower limit on $\lambda$ suggests, surprisingly, that the mean free path of the hot electrons is of the same order as that of cold electrons. The $\alpha$ was further improved when two additional gates were employed in the emitter region to stop completely the edge current.

\section{LO phonon emission}

The studies discussed in the present and the next sections were done on the structure shown in Fig.1c, where the continuous gates of the device presented in Fig.1a were replaced by pairs of gates. The circular layout of the collectors was designed to provide information on the spatial distribution of the collected electrons and the replacement of a continuous gate by pairs of gates was motivated by the flexibility it provides in working with either cold (low negative gates voltage, where the opening are not pinched off) or hot (large negative gates voltage, where the opening are depleted of carriers) electrons. The nominal sizes of the openings were usually $180 \mathrm{~nm}$ and the radius of the 'collector circle' varied between 1 and 2 $\mu \mathrm{m}$. Typical results for the collector current (dashed lines) and the transfer ratio (solid lines) as a function of the injection voltage $V_{E B}$ are depicted in Fig.4. The two sets of curves correspond to different emitter gates voltages $V_{G E}=-1.2$ and $-1.24 \mathrm{~V}$, and a fixed collector barrier height of approximately $20 \mathrm{meV}$ above Fermi level in the base. For small emitter biasing, its opening is pinched off. An increased voltage lowers the emitter barrier below the Fermi energy in the emitter, leading to a finite injection current. The collector current starts as soon as the injection energy exceeds the collector barrier height proving ballistic transport. The transfer ratio, $\alpha$, reaches a sharp maximum at $V_{E B} \approx 36 \mathrm{mV}$ then drops down to a minimum at $V_{E B} \approx 55 \mathrm{mV}$, reaches a second peak at $V_{E B} \approx 70-75 \mathrm{mV}$ and a third one 
at approximately $110 \mathrm{mV}$. The value of $\alpha$ agrees well with ballistic transport in the solid angle covered by the collector (approximately $30^{\circ}$ ), implying non or very weak large-angle scattering. The three distinct peaks in $\alpha$ lie in the vicinity of one, two and three phonon energy quanta above the Fermi energy in the base (i.e. $V_{E B}=36,72$ and $108 \mathrm{mV}$ ) and result from one, two, and three sequential LO phonons emission by the injected hot electrons.

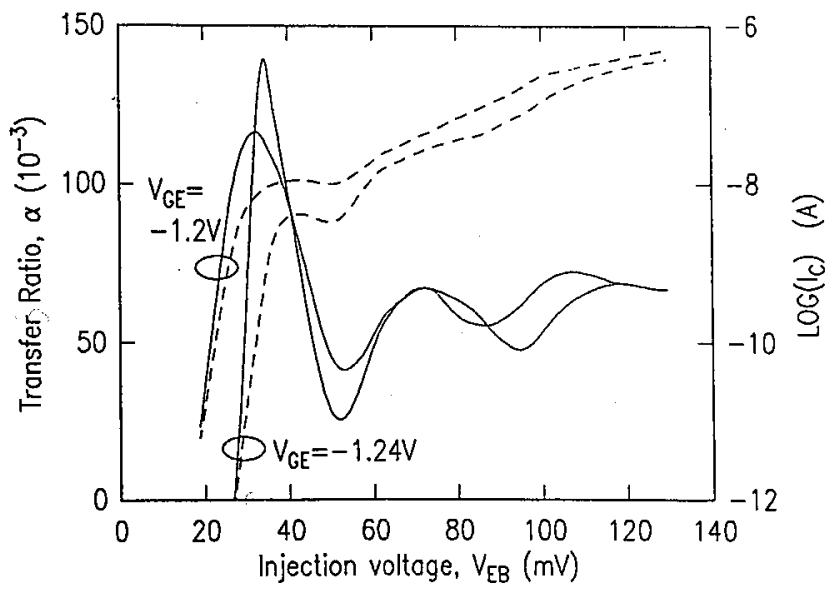

Fig.4. The oscillations in the transfer ratio (solid lines) and the collector current (dashed lines) vs. the injection voltage for two values of emitter gates voltages measured at $T=4.2 \mathrm{~K}$. Notice the negative differential transconductance $\left(d I_{C} / d V_{E B}\right)$. The collector barrier is fixed at approximately $20 \mathrm{meV}$ above the Fermi energy in the base.

At $4.2 \mathrm{~K}$, the occupation number of LO phonons is negligible and the phonon emission mfp for an electrons with energy $E=43 \mathrm{meV}\left(E_{F}+36 \mathrm{meV}\right)$ is approxImately $500 \AA[7]$, much shorter then the separation between the emitter and the collector. We thus expect folding of all collected electron energies to the range between 0 and $36 \mathrm{meV}$ above the Fermi energy in the base. Electrons having an energy $E+n \times 36 \mathrm{meV}$ above $E_{F}$ in the base, where $n=0,1,2, \ldots$, and $E<36$ $\mathrm{meV}$, emit $n$ phonons sequentially and relax to an energy $E$, as demonstrated by the oscillations in $\alpha$ shown in Fig.4.

The results described above do not show evidence of significant scattering below $36 \mathrm{meV}$. This is a surprising result since electron-hole excitation (in the conduction band) and electron-plasmon scattering are expected to yield a relatively short $\mathrm{mfp}[8,9]$. The first mechanism dominates the scattering at energies close to the Fermi energy while the latter is operative above some critical energy relative to $E_{F}$. For our 2DEG carrier concentration the threshold wave vector, $k_{C}$, for plasmon scattering is expected at $k_{C} \approx 1.85 k_{F}$ ( $k_{F}$ is the Fermi wave vector) or $E_{C} \approx 17 \mathrm{meV}$ above the Fermi energy [8]. A comparable threshold is also given in [9] for slightly higher carrier concentration $\left(3 \times 10^{11} \mathrm{~cm}^{-2}\right)$. Thus, for energies above this threshold, the inelastic mfp is predicted to be $200-250 \AA$ while below 
$E_{C}$ it is expected to be roughly of the order of $1000 \AA[8,9]$, an order of magnitude shorter than the device's size. We can not account for this discrepancy with theory.

\section{Beam steering and focusing}

The angular distribution of the hot electrons injected from a point source emitter (Fig.1c) is shown in Fig.5. If the scattering length had been much shorter

\section{ANGULAR DISTRIBUTION}

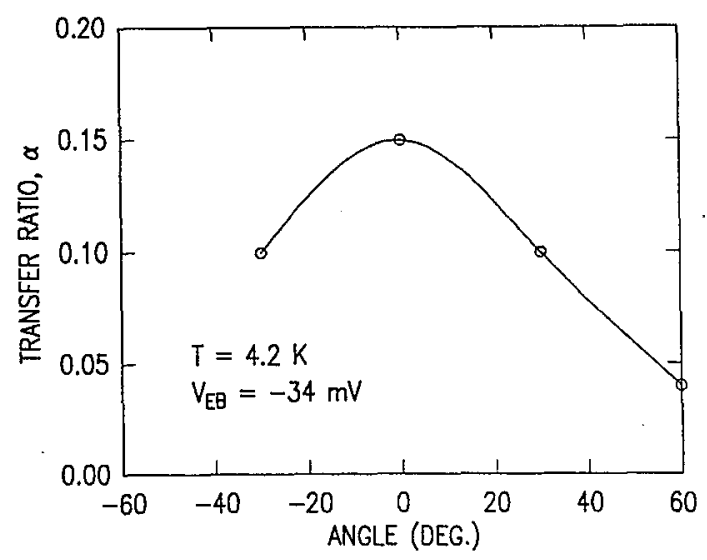

Fig.5. The transfer ratio in the various collectors as a function of the angle measured from the symmetry axis of the emitter.

than the distance between emitter and collectors, one would have expected equal transfer ratio to all four collectors. Since the motion here is quasi ballistic, a pronounced preference to the forward direction is observed. In the limit where the wavelength is longer than the emitter opening, and the horn flares adiabatically, theory predicts a $\cos ^{2}(\theta)$ dependence of $\alpha$ on the angle $\theta$. The results in Fig.5 confirm this prediction. The directionality of the point emitter and the dependence of that direction of the electrostatic profile created by the gates provide a novel method for steering the electron beam. An increased voltage on the left emitter gate for example will tilt the symmetry axis of the emitter and hence steer the electron beam to the right. The results of such an experiment are depicted in Fig.6. There we show the transfer ratio in the $30^{\circ}$ collector as a function of the difference between the voltage on the two emitter gates. $\alpha$ indeed vary between 0.05 and 0.25 in the expected direction. The high transfer ratio for $V_{G L}-V_{G R}=-0.2 \mathrm{~V}$ is somewhat surprising and might result from focusing effect in the emitter.

The focusing of electron beam is achieved by a spatial modulation of the electrostatic potential in transport region using a biconcave shape of the gate shown in Fig.1d. The corresponding "Snell's law" for ballistic electrons crossing 


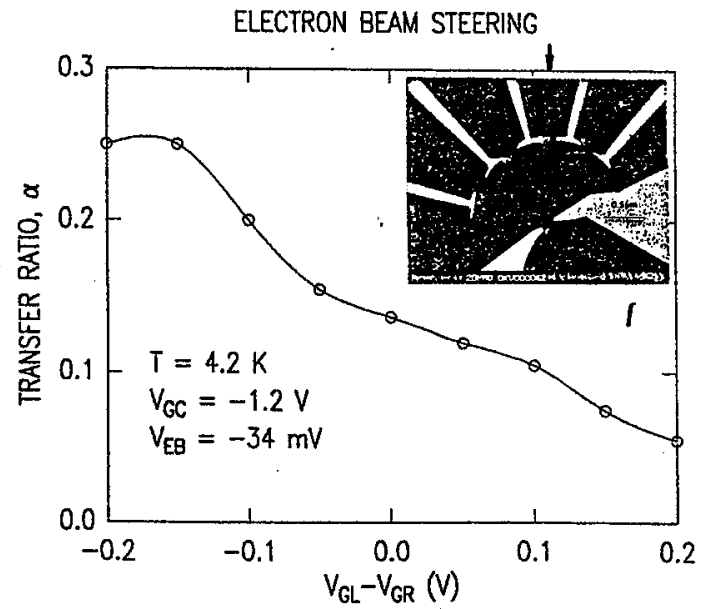

Fig.6. Electron beam steering. The transfer ratio in the collector at $30^{\circ}$ relative to the emitter's symmetry axis as a function of the voltage difference applied to the two emitter gates.

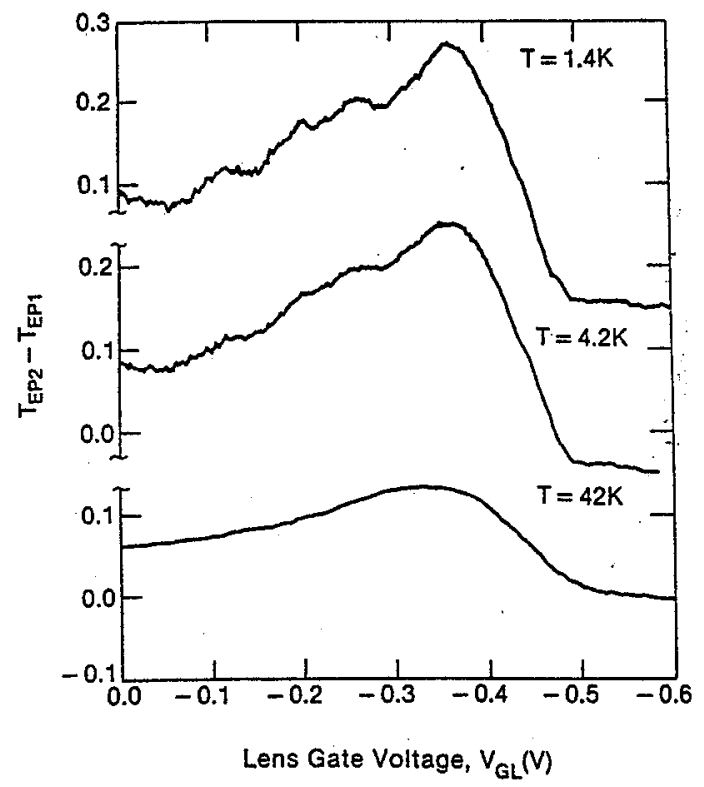

Fig.7. Electron beam focusing. The difference between the transmission coefficients to the two voltage probes $\mathrm{P} 1$ and $\mathrm{P} 2$ vs. the voltage applied to the lens gate. 
the boundary of different potentials is:

$$
\frac{\sin \theta_{1}}{\sin \theta_{2}}=\left(1-\frac{\Phi}{E_{F}}\right)^{\frac{1}{2}}
$$

where $\Phi$ is a potential difference, $E_{F}$ is Fermi energy, $\theta_{1}$ and $\theta_{2}$ are incident and refraction angles respectively.

Note that for $\Phi>0, \theta_{2}>\theta_{1}$, that implies necessity of biconcave lens to focus electrons. Applying a negative voltage on the gate $V_{G L}$ the potential $\Phi$ can be varied between zero and values larger than $E_{F}$, resulting in the change of the focal length between the values of $R$ (lens radius) and infinity. The requirement of equal distances from the object to the lens and from the image to the lens yields:

$$
L=\frac{2 R}{1-\left(1-\frac{\Phi}{E_{F}}\right)^{\frac{1}{2}}}+W,
$$

where $L$ is the distance from object to image and $W$ is the lens width. For the present experiment this implies $\Phi=\frac{2}{3} E_{F} \approx 6.7 \mathrm{meV}$.

The focusing by the lens should be reflected in maximum of current transmitted from the emitter to the collector for a certain value of negative bias applied to the gate. Figure 7 shows the variation of the difference between transmission coefficients to the voltage probes $P_{1}$ and $P_{2}$ vs. voltage applied to the lens gate (relationship between transition coefficients and measured voltage difference between $P_{1}$ and $P_{2}$ is given in details in [6]). A clear maximum is observed at $V_{G L} \approx-0.37$ $\mathrm{V}$ in a good agreement with theoretical estimate $(-0.38 \mathrm{~V})$. The transmission maximum in Fig.7 is less pronounced at higher temperatures and disappears completely at $77 \mathrm{~K}$, confirming the ballistic nature of the observed effect. The further verification of ballisticity is provided by measurements in presence of magnetic field. The focusing peak decreases and finally vanishes (Fig.8,), when a magnetic field perpendicular to a plane of 2DEG is applied and reaches $\sim 700 \mathrm{G}$, where the cyclotron orbit diameter approaches the distance between emitter and collector $(\sim 2.5 \mu \mathrm{m})$.

\section{Summary}

Quasi ballistic transport in a 2DEG was explored and tunneling through an electrostatic barrier induced by metallic gates on top of the heterostructure was demonstrated. For energies above $36 \mathrm{meV}$ we observed the LO phonon emission, leading to a short mfp. At energies below the phonon energy we find a mfp of the order of $2 \mu \mathrm{m}$, about an order of magnitude longer than expected theoretically for electron interactions. The angular distribution of hot electrons injected from a point source was shown to be collimated and could be steered by controlling the electrostatic profile of the injector. In addition we demonstrated operation of electrostatic lens which opens a new branch in semiconductor physics, namely, electron-optics in solids. 


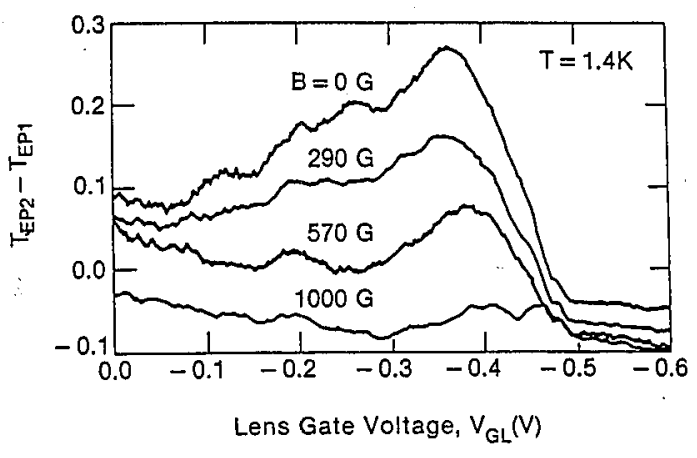

Fig.8. Effect of magnetic field on focusing. The difference between the transmission coefficients to the two voltage probes, $\mathrm{P} 1$ and $\mathrm{P} 2$ vs.voltage applied to the lens gate for various values of a magnetic field pexpendicular to the plane of the 2DEG.

\section{Acknowledgment}

We thank L.Osterling for his contribution in the MBE growth.

\section{References}

[1] F. Capasso, S. Datta, Physics Today 43, No2, 74 (1990), and references cited therein.

[2]. H. Smith, H. Craighead, Physics Today 43, No2, 24 (1990), and references cited therein.

[3] A. Palevski, M. Heiblum, C.P. Umbach, C.M. Knoedler, R. Koch, A. Broers, Phys. Rev. Lett. 62, 1776 (1989).

[4] A. Palevski, M. Heiblum, C.P. Umbach, Appl. Phys. Lett. 55, 1421, (1989).

[5] U. Sivan, M. Heiblum, C.P. Umbach, Phys. Rev. Lett. 63, 992 (1989).

[6] U. Sivan, M. Heiblum, C.P. Umbach, H. Strickman, Phys. Rev. B 41, 7937 (1990).

[7] W. Fawcett, A.D. Boardman, S. Swain, J. Phys. Chem. Solids 31, 1963 (1970).

[8] R. Jalabert, S. Das Sarma, unpublished (1989).

[9] P.H. Hawrylak, G. Eliasson, J.J. Quinn, Phys. Rev. B, 37, 10187 (1988), and references cited therein. 